Jorge Avelino Bento Maria Lúcia Lebrão ${ }^{1}$
${ }^{1}$ Faculdade de Saúde Pública, USP. Av. Dr. Arnaldo 715, Cerqueira Cesar. 01246-904 São Paulo SP.jabento@usp.br

\title{
Suficiência de renda percebida por pessoas idosas no Município de São Paulo/Brasil
}

\author{
Sufficiency of income perceived by the elderly \\ in the city of São Paulo /Brazil
}

\begin{abstract}
During the course of their lives elderly Brazilians have witnessed a country with major social contradictions, where the differences between individuals in relation to the factors involved in treatment and understanding of their needs are many. In 2000, factors associated with the perception of income sufficiency of elderly people (60 years and over) in São Paulo were analyzed, in an attempt to understand a few of these aspects. A crosssectional epidemiological study was conducted by interviews with 2,143 elderly people (60 years and over). Of these, 1,300 needed no help in answering the questionnaire and scored 13 or more points on the Mini Mental State Examination. They had salaried work at some stage, had some form of income and this matched the amount of income sources reported. Thirty-one percent of the elderly perceived their income as sufficient. Those with income above poverty levels had higher odds of satisfactory perception. Regardless of income, they were associated with: having predominantly worked in non-physical labor; started work at 14 years or older; having private health insurance; more than one source of income; not suffering privation in the first 15 years of life; being 70 or older; enjoying good health and autonomy in daily activities without assistance.
\end{abstract}

Key words Aging, Socioeconomic factors, Income, Financial sufficiency, SABE study
Resumo Os idosos brasileiros presenciaram, em seu curso de vida, um país com grandes contradições sociais e as diferenças no tocante aos fatores envolvidos no atendimento e entendimento de suas necessidades são muitas. Para tentar compreender alguns desses aspectos foram analisados os fatores associados à percepção de suficiência de renda dos idosos da cidade de São Paulo, em 2000. Estudo epidemiológico de corte transversal, realizado a partir da entrevista com 2143 idosos do Estudo SABE no qual foram identificados 1300 que não precisaram de ajuda para responder o questionário, obtiveram 13 pontos ou mais no Mini Exame de Estado Mental, trabalharam com remuneração em algum momento, tinham algum tipo de renda e que esta coincidisse com a quantidade de fontes de renda informada. Perceberam como suficiente sua renda, 31\% deles. Aqueles com renda acima das medidas de pobreza analisadas apresentaram maiores chances de percepção satisfatória e, independente da renda, estiveram associados: trabalho predominante em atividades não físicas, início da atividade laboral com 14 anos ou mais, ter plano privado de saúde, ter duas ou mais fontes de renda, não privação alimentar nos 15 anos iniciais de vida, ter 70 anos ou mais, ter boa saúde e autonomia na realização de atividades da vida diária.

Palavras-chave Envelhecimento, Fatores socioeconômicos, Renda, Suficiência financeira, Estudo $S A B E$ 


\section{Introdução}

Com o avanço da população idosa o processo de envelhecimento das sociedades passou a ser, cada vez mais, reconhecido em diversos encontros nacionais e internacionais ${ }^{1}$. O Plano Internacional de Ação sobre Envelhecimento (PIAE), elaborado na $2^{\text {a }}$ Conferência Mundial, trouxe à tona a discussão de que a intersetorialidade entre as áreas da saúde, economia, mercado de trabalho e educação seria muito importante para garantir a efetividade das políticas voltadas ao envelhecimento ${ }^{2}$.

O Brasil, apesar de significativos avanços nos aspectos sociais e econômicos, ainda é um país com grandes desigualdades sociais. Os idosos brasileiros presenciaram, durante seu curso de vida, um país com grandes contradições sociais. Os idosos latino-americanos, de uma forma geral, foram expostos, quando jovens, a situações que limitaram sua ascensão social ${ }^{3}$.

As diferenças verificadas quanto à real situação de pobreza de uma determinada população, entre elas os idosos, decorrem das inúmeras medidas e conceitos existentes sobre como, efetivamente, classificar alguém como pobre.

A pobreza é um problema multidimensional, sendo que suas causas variam dependendo da idade, gênero, cultura e outros fatores sociais e econômicos ${ }^{4}$. As comparações entre os resultados encontrados nos diversos estudos sobre o tema possuem problemas metodológicos e conceituais, entre os quais as diferentes medidas de linhas de pobreza e de bem-estar existentes ${ }^{5-7}$.

Há limitações em considerar as medidas de pobreza convencionais (ex. linhas de pobreza) como indicadoras fiéis da real condição de vida, principalmente dos idosos; pois estes possuem hábitos e necessidades especificos ${ }^{8}$. Se as medidas atuais de pobreza fossem complementadas com as análises subjetivas, um conjunto maior de informações seria obtido? .

Acrescente-se a isso que a satisfação do que se entende como necessidade mínima, os hábitos de consumo e as preferências das pessoas podem variar conforme o ambiente em que estão inseridas. Apesar de ser possível definir a quantidade de calorias mínimas que uma determinada pessoa precisaria consumir, por exemplo, em um dia, o que ela irá consumir e em que quantidade, dependerá, entre outras coisas, de suas preferências de consumo, que são, ainda, pouco conhecidas ${ }^{10}$.

Para Amartya Sen deve-se ir além do pensamento utilitário para satisfazer prazeres ou desejos dos indivíduos. A limitação de renda não seria o único fator que impediria as pessoas de poder ou não concretizar algo, pois as características físicas e sociais adquiridas afetariam significativamente suas vidas ${ }^{11}$.

Entende-se que seria importante a elaboração de estudos que pudessem trazer à tona informações quanto às diferenças existentes entre os indivíduos no tocante aos fatores envolvidos no atendimento de suas necessidades. Através dos idosos, que vivenciaram diversos fatores e circunstâncias em sua trajetória de vida, novos subsídios poderão surgir no tocante ao entendimento dessa questão, contribuindo, deste modo, para garantir aos idosos o direito á autorrealização, à saúde, seguridade e participação ativa na vida econômica, social, cultural e política do ambiente em que estão inseridos ${ }^{12}$.

Este trabalho tem por objetivo desenvolver a análise dos fatores associados à percepção de suficiência de renda dos idosos do município de São Paulo, em 2000. Acredita-se que, ao identificá-los, os resultados possam ser úteis para o entendimento de aspectos envolvidos no atendimento de algumas necessidades da população idosa, assim como no desenvolvimento de outros estudos que continuem contribuindo para a melhoria de sua qualidade de vida.

\section{Material e métodos}

O presente trabalho foi um estudo transversal realizado com dados provenientes do estudo Saúde Bem-Estar e Envelhecimento - SABE, realizado no município de São Paulo. Este foi um estudo multicêntrico, coordenado pela Organização Pan-Americana de Saúde, realizado em sete centros urbanos na América Latina e Caribe (Argentina, Barbados, Brasil, Chile, Cuba, México e Uruguai), incluindo o município de São Paulo, durante o período de outubro de 1999 a dezembro de 2000. No município de São Paulo o trabalho foi coordenado pela Faculdade de Saúde Pública, incluiu uma amostra de 2.143 indivíduos de 60 anos e mais. A amostra final foi alcançada por meio da somatória de uma amostra probabilística de 1568 idosos e uma sobreamostra formada por 575 indivíduos de 75 anos ou mais, correspondente ao acréscimo efetuado para compensar a mortalidade desse grupo etário. O processo de amostragem foi realizado pelo método de conglomerados em dois estágios sob o critério de partilha proporcional ao tamanho, sendo o primeiro correspondente ao sorteio dos setores censitários e o segundo ao sorteio do domicílio. Um cadastro permanente de 72 setores censi- 
tários, existente no Departamento de Epidemiologia da Faculdade de Saúde Pública, foi considerado a amostra de primeiro estágio. Essa amostra foi tomada do cadastro da Pesquisa Nacional por Amostra de Domicílios (PNAD), 1995, composto por 263 setores censitários sorteados sob o critério de probabilidade proporcional ao número de domicílios. O número mínimo de domicílios sorteados no segundo estágio foi aproximado para 90. A complementação da amostra de pessoas de 75 anos e mais foi realizada por meio da localização de moradias próximas aos setores selecionados ou, no máximo, dentro dos limites dos distritos aos quais pertenciam os setores sorteados. Cada questionário teve um peso calculado de acordo com o setor censitário de que faz parte (peso = 1/f) e, para os questionários realizados com indivíduos em domicílios que foram selecionados, porém não sorteados (faixa etária 75 anos e mais), o cálculo do peso foi realizado de acordo com a relação da população de idosos (de ambos os sexos) nessas faixas etárias, residentes no Município de São Paulo em 1998, e o número de idosos nessas mesmas faixas encontradas na amostra final do estudo ${ }^{13}$.

A coleta de dados foi feita na residência dos idosos, por entrevistadores treinados, e incluiu a aplicação de questionário estruturado contendo questões relativas às condições sociodemográficas, condição econômica, saúde geral, acesso e uso de serviços, redes de apoio familiar e uma bateria de exames e testes antropométricos.

A variável dependente deste estudo foi a autopercepção de suficiência de renda para despesas básicas medida por meio da seguinte questão: "O $\mathrm{Sr}$ (a) considera que tem dinheiro suficiente para cobrir suas necessidades de vida diária?".

As variáveis independentes foram as seguintes: sociodemográficas (idade, raça/cor, estudo, escolaridade e história laboral), condições de saúde (autopercepção de saúde, dificuldades em Atividades Básicas da Vida Diária (ABVD), dificuldades em Atividades Instrumentais da Vida Diária (AIVD), autopercepção do estado nutricional, consumo de três ou mais refeições por dia), e condições econômicas (autopercepção de situação econômica, tipo e fontes de renda disponíveis, situação em relação a algumas medidas de renda, posse de plano de saúde e modo de pagamento de despesas diárias).

As medidas de renda utilizadas foram: renda total do idoso maior que o valor total da cesta básica de alimentos para consumo de sua família, renda total do idoso maior que o valor da cesta básica de alimentos e outros itens necessá- rios para o consumo de sua família, renda por dependente maior que $1 / 2$ salário mínimo e renda por dependente maior que $1 / 4$ do salário mínimo vigente no período da entrevista.

A variável renda maior que o valor da cesta básica de alimentos foi obtida através da divisão do valor total da renda do idoso pelo valor da cesta básica de alimentos da família. Neste caso, idosos cujo resultado da renda fosse igual ou superior a 1 foram classificados como detentores de renda maior que o valor da cesta básica de alimentos.

Para identificar o valor da cesta básica de alimentos foram utilizados dados de consumo mínimo de calorias, conforme preconizado pela FAO/OMS ${ }^{14}$, em 2000. Ou seja, estimou-se o consumo necessário para os idosos e para cada uma das pessoas com quem residia, segundo a unidade equivalente de consumo para cada pessoa. E, através da pesquisa de preço da cesta básica divulgada pelo DIEESE ${ }^{15}$, estimou-se, conforme o mês em que a entrevista havia sido realizada, qual seria o valor da cesta básica de alimentos para cada uma das famílias, assim como para o idoso que morava só ou com parceiro.

Durante o ano de 2000 o valor médio da cesta básica, segundo o DIEESE, foi de R\$113,63. Ao se calcular a renda familiar média necessária para suprir o conjunto de necessidades dos indivíduos na residência dos idosos estudados, conforme unidade equivalente de caloria de cada pessoa e tendo como referência o preço da cesta básica, chegou-se a um valor médio de $\mathrm{R} \$ 287,22$.

A renda do idoso maior que a cesta básica de alimentos e de itens necessários para sua família foi obtida através de procedimento semelhante ao anterior. Entretanto, com o acréscimo do custo dos itens não alimentares determinado pela utilização do coeficiente de Engel ${ }^{16,17}$, obtido através da relação entre despesas alimentares e despesa total. O resultado encontrado foi somado ao da cesta básica de alimentos, gerando o valor de uma das diversas medidas de linhas de pobreza existente ${ }^{9,10,18-20}$.

Os dados da Pesquisa de Orçamentos Familiares (POF) 2002-2003²1, foram utilizados como referência para definir a distribuição de renda entre despesas alimentares e não alimentares, resultando no coeficiente de Engel de aproximadamente 0,4025. Desse modo, o valor médio necessário para atender às necessidades básicas da vida diária da família em que o idoso estava inserido seria de $\mathrm{R} \$ 713,58$.

Foram considerados idosos situados abaixo da linha de indigência ou abaixo da linha de po- 
breza aqueles que apresentaram renda total por dependente menor que $\mathrm{R} \$ 37,75$ ou $1 / 4$ do salário mínimo vigente ou menor que $\mathrm{R} \$ 75,50$ ou $1 / 2$ do salário mínimo vigente, respectivamente. Em 2000, o salário mínimo (SM) teve três valores: $\mathrm{R} \$ 136,00$ entre janeiro e março, $\mathrm{R} \$ 150,00 \mathrm{em}$ abril e $\mathrm{R} \$ 151,00$ a partir de maio ${ }^{22}$. As rendas citadas com valores idênticos aos do SM, nos primeiros quatro meses, foram corrigidas para $o$ valor vigente em maio e as rendas informadas que não possuíam valor idêntico ao SM vigente em maio foram corrigidas em 5,81\%, valor do reajuste da aposentadoria em $01 / 06 / 2000^{23}$.

Neste estudo foram excluídos os idosos que nunca tiveram trabalho remunerado $(\mathrm{n}=142)$; que não possuíam nenhum tipo de renda (n =141); que responderam ao questionário com ajuda de proxy $(n=183)$, que apresentaram declínio cognitivo no momento da entrevista - pontuação menor do que 13 pontos no teste Mini Exame do Estado Mental - MEEM ( $\mathrm{n}=192)$, pessoas com divergências quanto ao relato do número e do tipo de fontes de rendas e os valores de renda informados $(\mathrm{n}=185)$, restando 1300 indivíduos para análise.

Ressalte-se que, conforme Lebrão e Laurenti $^{23}$, a utilização do MEEM, desenvolvido por Folstein et al. ${ }^{24}$, ocorreu através de uma versão modificada e validada no Chile, por Icaza e Albala, em $1999^{25}$.

A análise de dados foi feita por meio da descrição dos dados e testes de associação. Utilizouse o teste de Rao-Scott ${ }^{26}$ para medir a associação entre variáveis categóricas. Todas as variáveis com $\mathrm{p}<0,20$ foram incluídas no modelo de regressão logística múltipla pelo método backwards. O ajuste final dos modelos foi avaliado por meio do teste de Hosmer-Lemeshow para amostras complexas $^{27}$. Todas as análises foram ponderadas e realizadas por meio do software Stata 11.0 (STATA Corp., College Station, Estados Unidos), utilizando-se o comando "survey" para análise de amostras complexas.

Este trabalho foi aprovado pelo Comitê de Ética em Pesquisa da Faculdade de Saúde Pública da Universidade de São Paulo e pela Comissão Nacional de Ética em Pesquisa. Todos os participantes e entrevistadores assinaram o consentimento livre e esclarecido no momento da entrevista.

\section{Resultados}

A população investigada de 1.300 idosos representava o universo populacional de 524.757 pes- soas de 60 anos e mais residentes na cidade de São Paulo em 2000.

A proporção de idosos que relatou possuir dinheiro suficiente para cobrir suas necessidades da vida diária foi de $31,0 \%$, sendo que na análise bivariada, idosos mais velhos (70 anos e mais) apresentaram maior satisfação com a renda disponível quando comparados com idosos mais jovens (60 a 69 anos), 33,9 e 29,2\%, respectivamente (Tabela 1).

Idosos que se autodenominaram da raça/cor branca ou asiática possuíam percepção de suficiência de renda maior do que os autodenominados, entre outros, negros, mulatos e indígenas (Tabela 1). Identificou-se que $18,2 \%$ dos idosos nunca foram à escola e que somente $23,5 \%$ possuíam cinco ou mais anos de estudo. O idoso que relatou ter frequentado escola, sem especificação de tempo, apresentou risco de chance maior de estar satisfeito com sua disponibilidade de renda do que entre os idosos que nunca a frequentaram e, entre os idosos que mais estudaram (oito anos e mais), a maioria percebeu sua renda como suficiente (Tabela 1). O nível de satisfação com a renda disponível entre os idosos mais escolarizados foi sempre maior, independente da categorização que se faça.

$\mathrm{Na}$ análise de algumas características das condições socioeconômicas dos idosos, em seus primeiros quinze anos de vida, constatou-se que o nível de satisfação com a renda disponível foi maior entre os idosos que relataram dificuldades financeiras nessa fase da vida do que nos que relataram ter passado fome. $\mathrm{O}$ diferencial verificado foi que, na análise univariada, os idosos que nunca passaram fome possuíam $83 \%$ de chances a mais de estarem satisfeitos com sua renda, bem acima do verificado entre os que tiveram uma boa situação econômica (Tabela 1).

Quanto mais cedo o idoso começou a trabalhar, menos satisfatória foi sua percepção de suficiência de renda (Tabela 1). A média de idade de início de trabalho dos homens foi de 12,1 anos e das mulheres 15,6 anos

Quanto ao tipo de atividade laboral desenvolvido, $55,2 \%$ das mulheres e $44,8 \%$ dos homens relatou ter exercido atividades predominantemente físicas. Verificou-se que idosos que não trabalharam predominantemente em atividades físicas, ou seja, predominantemente intelectual e parcialmente física com intelectual tiveram mais chances de estarem satisfeitos com a sua renda (Tabela 1).

Ao serem questionados se a atividade referida foi a que exerceu predominantemente em sua 
vida profissional, 59,9\% das mulheres e $67,9 \%$ dos homens responderam afirmativamente; apresentando sentimento de satisfação maior do que aqueles que apresentaram maior rotatividade (Tabela 1).

Observou-se que a maioria dos idosos tinha apenas uma fonte de renda $(57,7 \%$ entre os homens e $69,2 \%$ entre as mulheres), sendo a percepção de suficiência de renda desse grupo menor do que os que possuíam mais de uma fonte (Tabela 2).

Independente da medida de renda utilizada, idosos com menor rendimento têm níveis bem menores de satisfação quanto à sua percepção de suficiência de renda (Tabela 2).

A capacidade de arcar com a totalidade de despesas diárias como luz, água, telefone, alimentos, vestimentas e gastos médicos foi identificada como um importante fator na contribuição para uma melhor avaliação da situação de renda, pois idosos em tais condições apresentaram percepção de suficiência de renda bem acima dos demais (Tabela 2).

Idosos com melhor condição de saúde (excelente, muito boa ou boa) apresentaram maior nível de satisfação com sua renda do que idosos em pior estado (Tabela 3). Quando analisados os idosos com renda total superior ao valor da cesta básica familiar, os resultados foram semelhantes, ou seja, 46,2\% dos idosos com melhor saúde consideraram sua renda suficiente ao contrário de 33,2\% dos idosos que se declararam em pior estado de saúde.

Os idosos que dispunham de seguro de saúde privado apresentaram maior chance de estar satisfeitos com sua renda do que os que não o possuíam (Tabela 3). Entre os que dispunham do seguro privado, $46,9 \%$ relataram ter boa condição de saúde e $48,6 \%$ possuíam sua renda total maior que o valor da cesta básica de alimentos necessária para o conjunto de sua família. Apesar disso, a correlação entre possuir renda acima do mínimo necessário para o consumo de alimentos e possuir o seguro privado não foi significativa (índice de 0,2347).

No primeiro modelo de regressão logística multivariada (Tabela 4), considerou-se como variável de renda aquela que media a proporção de idosos com renda total superior ao mínimo necessário para atendimento das necessidades

Tabela 1. Distribuição dos idosos segundo variáveis sociodemográficas, condição pregressa de vida e percepção de suficiência de renda, município de São Paulo, 2000 ( $\mathrm{n}=1.300)$.

\begin{tabular}{|c|c|c|c|c|c|c|c|}
\hline \multirow[b]{2}{*}{ Variável } & \multirow[b]{2}{*}{ Categoria } & \multirow[b]{2}{*}{$\%$ total } & \multicolumn{5}{|c|}{ H30 - Considera renda suficiente } \\
\hline & & & $\%$ & $\mathbf{p}$ & $\beta$ & odds & $95 \%$ IC \\
\hline \multirow[t]{2}{*}{ Idade } & 60 a 69 & 61,9 & 29,2 & - & - & - & - \\
\hline & 70 anos e mais & 38,1 & 33,9 & 0,0986 & 0,22 & 1,25 & $0,96-1,62$ \\
\hline \multirow[t]{2}{*}{ raça/cor } & não brancAsia ${ }^{a}$ & 26,4 & 19,3 & - & - & - & - \\
\hline & brancAsia & 73,6 & 35,2 & 0,0000 & 0,82 & 2,27 & $1,60-3,23$ \\
\hline \multirow[t]{2}{*}{ foi a escola } & não & 18,2 & 16,9 & - & - & - & - \\
\hline & $\operatorname{sim}$ & 81,8 & 34,1 & 0,0000 & 0,93 & 2,54 & $1,73-3,74$ \\
\hline \multirow[t]{4}{*}{ escolaridade } & nenhuma & 18,2 & 16,9 & - & - & - & - \\
\hline & 1 a 4 anos & 58,3 & 28,7 & - & 0,68 & 1,98 & $1,33-2,95$ \\
\hline & 5 a 8 anos & 9,3 & 38,5 & - & 1,12 & 3,08 & $1,88-5,04$ \\
\hline & mais de 8 anos & 14,2 & 53,3 & 0,0000 & 1,72 & 5,60 & $3,34-9,39$ \\
\hline \multirow[t]{2}{*}{ Boa situação econômica ${ }^{b}$} & não & 71,2 & 29,6 & - & - & - & - \\
\hline & $\operatorname{sim}$ & 28,8 & 34,4 & 0,1853 & 0,22 & 1,25 & $0,90-1,74$ \\
\hline \multirow[t]{2}{*}{ Alimentou-se bem ${ }^{b}$} & não & 20,6 & 21,5 & - & - & - & - \\
\hline & $\operatorname{sim}$ & 79,4 & 33,4 & 0,0005 & 0,60 & 1,83 & $1,31-2,56$ \\
\hline \multirow[t]{2}{*}{ Labor depois dos 14 anos $^{c}$} & não & 65,2 & 26,5 & - & - & - & - \\
\hline & $\operatorname{sim}$ & 34,8 & 39,4 & 0,0004 & 0,59 & 1,81 & $1,31-2,49$ \\
\hline \multirow[t]{2}{*}{ Labor não só físico ${ }^{\mathrm{d}}$} & não & 73,2 & 23,9 & - & - & - & - \\
\hline & $\operatorname{sim}$ & 26,8 & 50,4 & 0,0000 & 1,17 & 3,23 & $2,39-4,37$ \\
\hline \multirow[t]{2}{*}{ Atividade foi predominante ${ }^{e}$} & não & 36,2 & 25,6 & - & - & - & - \\
\hline & $\operatorname{sim}$ & 63,8 & 34,1 & 0,0031 & 0,41 & 1,50 & $1,15-1,96$ \\
\hline
\end{tabular}

Fonte: Estudo SABE. ${ }^{\mathrm{a}}$ Idosos que relataram não pertencer a raça/cor branca ou de origem asiática (japoneses, chineses, coreanos, etc.). Entre outros: negros, mulatos, indígenas, etc. ${ }^{b}$ Durante os primeiros 15 anos de vida. ${ }^{c}$ Início atividade laboral com 15 anos ou mais. ${ }^{\mathrm{d}}$ A tividade laboral predominante não envolvia somente atividade física, ou seja, física e mental ou somente mental.

e Atividade laboral exercida foi predominante durante toda a vida profissional. 
Tabela 2. Distribuição dos idosos segundo situação da renda e percepção de suficiência de renda, município de São Paulo, 2000.

\begin{tabular}{|c|c|c|c|c|c|c|c|}
\hline \multirow[b]{2}{*}{ Variável } & \multirow[b]{2}{*}{ Categoria } & \multirow[b]{2}{*}{$\%$ total } & \multicolumn{5}{|c|}{ H30 - Considera renda suficiente } \\
\hline & & & $\%$ & $\mathbf{p}$ & $\beta$ & odds & $95 \%$ IC \\
\hline \multirow[t]{2}{*}{ Tem duas ou mais fontes de renda } & não & 63,6 & 25,8 & - & - & - & - \\
\hline & $\operatorname{sim}$ & 36,4 & 40,0 & 0,0000 & 0,65 & 1,91 & $1,45-2,53$ \\
\hline \multirow[t]{2}{*}{ Renda total cesta alimento ${ }^{b}$} & não & 34,5 & 13,6 & - & - & - & - \\
\hline & $\operatorname{sim}$ & 65,5 & 40,2 & 0,0000 & 1,45 & 4,26 & $2,88-6,30$ \\
\hline \multirow[t]{2}{*}{ Renda total necessidades ${ }^{c}$} & não & 62,9 & 18,7 & - & - & - & - \\
\hline & $\operatorname{sim}$ & 37,1 & 51,9 & 0,0000 & 1,55 & 4,70 & $3,59-6,15$ \\
\hline \multirow[t]{2}{*}{ linha pobreza SM d } & não & 19,7 & 6,9 & - & - & - & - \\
\hline & $\operatorname{sim}$ & 80,3 & 36,9 & 0,0000 & 2,06 & 7,90 & $4,46-3,80$ \\
\hline \multirow[t]{2}{*}{ linha Indigência SM e } & não & 5,2 & 5,3 & - & - & - & - \\
\hline & $\operatorname{sim}$ & 94,8 & 32,4 & 0,0000 & 2,15 & 8,58 & $2,71-27,23$ \\
\hline \multirow[t]{2}{*}{ Paga sozinho as despesas diárias } & não & 65,3 & 25,0 & - & - & - & - \\
\hline & $\operatorname{sim}$ & 34,7 & 42,2 & 0,0000 & 0,78 & 2,18 & $1,64-2,90$ \\
\hline
\end{tabular}

Fonte: Estudo SABE. a incluídos idosos que não relataram número de dependentes. ${ }^{\mathrm{b}}$ Renda total idoso maior que o valor total da cesta de alimentos para consumo da família $(\mathrm{R} \$ 287,22)$. ${ }^{\mathrm{C}}$ Renda total idoso maior que o valor da cesta básica de itens necessários para sua família $(\mathrm{R} \$ 713,58) .{ }^{\mathrm{d}}$ Renda por dependente maior que $\mathrm{R} \$ 75,50$ ou $1 / 2$ do salário mínimo vigente. ${ }^{\mathrm{e}}$ Renda por dependente maior que $\mathrm{R} \$ 37,75$ ou $1 / 4$ do salário mínimo vigente.

Tabela 3. Distribuição dos idosos segundo características de condições de saúde e percepção de suficiência de renda, município de São Paulo, 2000.

\begin{tabular}{|c|c|c|c|c|c|c|c|}
\hline \multirow[b]{2}{*}{ Variável } & \multirow[b]{2}{*}{ Categoria } & \multirow[b]{2}{*}{$\%$ total } & \multicolumn{5}{|c|}{ H30 - Considera renda suficiente } \\
\hline & & & $\%$ & $\mathbf{p}$ & $\beta$ & odds & $95 \%$ IC \\
\hline \multirow[t]{2}{*}{ Relata ter melhor condição de saúde a } & não & 52,9 & 23,5 & - & - & - & - \\
\hline & $\operatorname{sim}$ & 47,1 & 39,4 & 0,0000 & 0,75 & 2,12 & $1,65-2,73$ \\
\hline \multirow[t]{2}{*}{ Dificuldade em realizar ABVD/AIVD ${ }^{b}$} & não & 21,2 & 20,3 & - & - & - & - \\
\hline & $\operatorname{sim}$ & 78,8 & 33,9 & 0,0001 & 0,70 & 2,01 & $1,41-2,87$ \\
\hline \multirow[t]{2}{*}{ Tem seguro/plano de saúde privado } & não & 60,3 & 21,5 & - & - & - & - \\
\hline & $\operatorname{sim}$ & 39,7 & 45,4 & 0,0000 & 1,11 & 3,04 & $2,30-4,02$ \\
\hline \multirow[t]{2}{*}{ Considera ser bem nutrido } & não & 7,3 & 20,0 & - & - & - & - \\
\hline & $\operatorname{sim}$ & 92,7 & 31,9 & 0,0392 & 0,62 & 1,86 & $1,02-3,40$ \\
\hline \multirow[t]{2}{*}{ Faz 3 ou mais refeições no dia } & não & 33,8 & 27,7 & - & - & - & - \\
\hline & $\operatorname{sim}$ & 66,2 & 32,7 & 0,1297 & 0,24 & 1,27 & $0,93-1,73$ \\
\hline
\end{tabular}

Fonte: Estudo SABE. ${ }^{a}$ Condição de saúde relatada como boa, muito boa ou excelente. ${ }^{b}$ Relatou que dispensa qualquer tipo de ajuda para realizar Atividades Básicas da Vida Diária (ABVD) ou Atividades Instrumentais da Vida Diária (AIVD)

básicas (alimentos e outras despesas) do conjunto da família. No segundo modelo (Tabela 5), a variável de renda utilizada foi aquela que identificava os idosos com renda per capita superior a $1 / 2 \mathrm{SM}$.

Os resultados do teste de Hosmer-Lemeshow (goodness-of-fit test), para amostras complexas, p-valor de 0,82 (modelo A) e 0,93 (modelo B), indicam que ambos estariam adequados ${ }^{28}$.

Tanto no modelo multivariado A quanto no B sete fatores em comum (em negrito nas Tabelas) estiveram associados à percepção de suficiência de renda dos idosos. A renda exerce papel significativo na percepção de sua suficiência, pois idosos situados em patamares acima das medidas utilizadas, nos dois modelos, tinham maiores chances de estar satisfeitos.

A diferença entre os idosos quanto à percepção de suficiência de renda ocorre quando se muda a medida utilizada: no modelo A, o diferencial foram aqueles que foram à escola e que exerceram atividade laboral, predominantemente em um mesmo ramo de atividade e no modelo $\mathrm{B}$, o surgimento da etnia como fator significativo, assim como a capacidade de pagar, sozinho, itens de despesas do dia-a-dia. 
Tabela 4. Modelo logístico multivariado A, percepção satisfatória de suficiência de renda entre idosos do município de São Paulo, 2000.

\begin{tabular}{lccccc}
\hline \multicolumn{1}{c}{ Variável } & odds & erro & t & p & 95\% IC \\
\hline Renda maior que necessidades família & 2,85 & 0,49 & 6,08 & 0,000 & $2,03-4,01$ \\
Trabalhou predominante não física & 1,69 & 0,28 & 3,13 & 0,002 & $1,21-2,36$ \\
Tem seguro/plano saúde privado & 1,77 & 0,28 & 3,63 & 0,000 & $1,30-2,42$ \\
Foi à escola & 1,35 & 0,29 & 1,41 & 0,162 & $0,89-2,06$ \\
Tem duas ou mais fontes de renda & 1,49 & 0,26 & 2,29 & 0,024 & $1,05-2,10$ \\
Saúde $^{a}$ & 1,55 & 0,21 & 3,20 & 0,002 & $1,18-2,04$ \\
Iniciou história laboral após os 14 anos & 1,42 & 0,24 & 2,01 & 0,047 & $1,00-1,99$ \\
Alimentou-se bem, primeiros 15 anos & 1,39 & 0,26 & 1,78 & 0,078 & $0,96-2,01$ \\
Atividade laboral foi predominante & 1,37 & 0,20 & 2,10 & 0,038 & $1,02-1,83$ \\
Tem 70 anos ou mais & 1,43 & 0,23 & 2,24 & 0,027 & $1,04-1,96$ \\
\hline
\end{tabular}

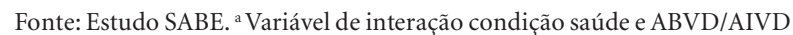

Tabela 5. Modelo logístico multivariado B, percepção satisfatória de suficiência de renda entre idosos do município de São Paulo, 2000.

\begin{tabular}{|c|c|c|c|c|c|}
\hline Variável & odds & erro & $\mathbf{t}$ & $\mathbf{p}$ & $95 \%$ IC \\
\hline Renda per capita maior $1 / 2$ S.M. & 4,06 & 1,25 & 4,54 & 0,000 & $2,20-7,48$ \\
\hline Trabalhou predominante não física & 1,83 & 0,30 & 3,62 & 0,000 & $1,31-2,54$ \\
\hline Tem seguro/plano privado de saúde & 1,89 & 0,29 & 4,18 & 0,000 & $1,40-2,56$ \\
\hline Branco ou asiático & 1,35 & 0,27 & 1,53 & 0,128 & $0,92-2,00$ \\
\hline Paga sozinho despesas vida diária & 1,39 & 0,22 & 2,05 & 0,043 & $1,01-1,91$ \\
\hline Tem duas ou mais fontes de renda & 1,49 & 0,25 & 2,39 & 0,018 & $1,07-2,08$ \\
\hline Saúde ${ }^{a}$ & 1,51 & 0,21 & 3,00 & 0,003 & $1,15-1,99$ \\
\hline Iniciou história laboral após os 14 anos & 1,43 & 0,24 & 2,12 & 0,036 & $1,02-1,99$ \\
\hline Alimentou-se bem, primeiros 15 anos & 1,31 & 0,23 & 1,51 & 0,134 & $0,92-1,86$ \\
\hline Tem 70 anos ou mais & 1,37 & 0,22 & 1,99 & 0,049 & $1,00-1,88$ \\
\hline
\end{tabular}

Fonte: Estudo SABE. a Variável de interação: boa condição saúde e dispensa ajuda ABVD/AIVD

\section{Discussão}

Os resultados mostram a importância da vida pregressa na situação presente dos indivíduos. Fatores ocorridos na fase inicial da trajetória de vida dos idosos foram importantes na avaliação de sua situação de renda, assim como fatores presenciados quando da realização da entrevista. Infere-se que ações, decisões e medidas adotadas durante o curso de vida dos idosos contribuíram para a análise da realidade em que estavam inseridos em 2000.

As questões relacionadas ao comportamento dos indivíduos junto ao ambiente em que estão inseridos são de grande importância para o entendimento de sua história de vida. As pessoas fazem a sua própria história, mas não de modo arbitrário, em circunstâncias escolhidas por eles mesmos e, sim, em circunstâncias diretamente dadas e herdadas do passado ${ }^{29}$.
Como já dito, de um modo geral, os idosos latino-americanos foram expostos a condições mais limitadas de ascensão social quando jovens ${ }^{3}$. Neste trabalho foi possível inferir que essa argumentação seria procedente ao se identificar, entre outros dados, o baixo nível educacional verificado entre os idosos analisados.

Os resultados sobre a escolaridade vão ao encontro do preconizado através da teoria do Capital Humano onde, entre outras características, pessoas com melhores níveis educacionais possuiriam maior chance de ter uma renda relativamente melhor do que as pessoas menos escolarizadas $^{30}$. Reforça-se que essa teoria não abrange somente o nível educacional como único investimento que os indivíduos realizam a fim de melhorar suas condições de vida. Os indivíduos investiriam em treinamento profissional, melhor condição de saúde como, por exemplo, cuidados preventivos em relação à sua saúde (procura de 
assistência médica ou cuidados alimentares), assim como atividades culturais e outros hábitos gerando externalidades melhores e positivas ${ }^{31,32}$.

Historicamente, o exercício de atividades predominantemente braçais estaria relacionado com atividades de baixa remuneração. Desse modo, não surpreende que idosos que trabalharam em atividades com maior nível de exigência intelectual tenham sido mais bem remunerados, o que ocasionaria, em principio, uma aposentadoria proporcionalmente maior e, eventualmente, o acúmulo de recursos durante sua vida laboral. Além disso, o exercício de uma atividade laboral predominante durante sua trajetória indicaria que esses idosos mantiveram maior estabilidade no ramo de atividade em que trabalhavam e que, provavelmente, obtiveram melhores resultados financeiros junto às atividades que desempenharam. Poder-se-ia inferir, nesses casos, que teriam tido, conforme a teoria do ciclo de vida ${ }^{33}$, uma propensão maior à ocorrência de poupança de recursos monetários.

A entrada no mercado de trabalho após os 14 anos indicaria que os idosos provavelmente pertenciam a famílias em melhores condições socioeconômicas e que, possivelmente, tiveram melhores condições de adquirir maior escolaridade do que aqueles que iniciaram atividade laboral com 14 anos ou menos. Além disso, reforçando a primeira hipótese, constatou-se melhor avaliação da situação de renda dos idosos que, em seus primeiros 15 anos de vida, nunca passaram por privação alimentar. São muitos os problemas que podem acometer as crianças inseridas precocemente no mercado de trabalho como, por exemplo, baixa escolaridade, problemas de saúde, exploração sexual, escravidão, etc. ${ }^{34}$.

A percepção de suficiência de renda tende a cair junto com a diminuição da renda real. Vários estudos identificaram que idosos com rendas mais baixas são significativamente mais propensos a relatar níveis inferiores de suficiência de ren$\mathrm{da}^{35-37}$. Pesquisa avaliando a percepção de satisfação com a renda de indivíduos classificados como $10 \%$ mais pobres e $10 \%$ mais ricos residentes em Andaluzia (Espanha) constatou que, entre os primeiros, apenas $2,2 \%$ estavam totalmente satisfeitos com sua renda, percentual bem inferior aos $21,5 \%$ da camada mais rica daquela localidade. Os autores acrescentam que a percepção de suficiência de renda dos mais pobres era influenciada negativamente pelo fato de seus rendimentos serem menores do que um determinado grupo de referência e que o mesmo não ocorria entre os mais ricos ${ }^{38}$.
A maior parte da renda dos idosos brasileiros é proveniente das aposentadorias e pensões e, tanto para homens quanto mulheres, sua importância tem crescido ao longo do tempo. Porém, ela é mais significativa para ambos os sexos na faixa etária de 60 a 64 anos. Já para as demais faixas, os benefícios previdenciários, entre eles os não contributivos (por exemplo, o BPC - Benefício de Prestação Continuada) seriam mais relevantes ${ }^{39}$.

Estudos indicam que idosos mais velhos tendem a superestimar sua satisfação com a renda ao subestimarem suas dificuldades financeiras ${ }^{35-37}$. Por serem mais velhos, esses idosos teriam melhores informações sobre sua condição de saúde, saberiam com mais precisão as descontinuidades a que estão suscetíveis e teriam maior experiência no gerenciamento de seus recursos, conforme seu nível de renda, em relação aos idosos mais jovens. Em complemento, haveria a teoria da dissonância cognitiva onde os idosos, ao presenciarem a diminuição de seus rendimentos, alterariam sua interpretação a respeito de quanto precisariam efetivamente para sobreviver satisfatoriamente ${ }^{35,36}$.

Debert discute a dupla vulnerabilidade às quais estariam sujeitos os idosos pertencentes a minorias étnicas (double jeopardy) ao serem, estes, vitimas de formas de discriminação e exclusão próprias das sociedades ocidentais. Entre os estudos que apresenta, há os que sugerem que as diferenças sociais existentes entre idosos negros e brancos seriam menores do que as existentes, por exemplo, entre brancos e negros de meia-idade ${ }^{40}$.

No Brasil, através dos dados da PNAD de 2005 , identificou-se que idosos brancos apresentavam uma renda $82 \%$ maior do que idosos negros e que, entre os adultos, os denominados brancos possuíam uma diferença a favor de $108 \%{ }^{41}$.

Stoler e Stoler relatam que problemas de saúde podem comprometer a avaliação dos idosos quanto à percepção de suficiência de renda, pois eles teriam presente a necessidade de uma reserva de recursos financeiros para fazer frente aos elevados gastos relacionados com sua condição de saúde ${ }^{37}$. Os problemas de saúde podem se refletir na capacidade de realização das atividades de vida diária e o inverso ocorreria entre os idosos que não precisavam de nenhum tipo de ajuda (ABVD ou AIVD), pois estes têm maiores chances de estar satisfeitos com sua renda do que aqueles que precisavam de ajuda para pelo menos uma dificuldade ${ }^{36}$.

As diferenças sociais verificadas e a trajetória de vida trilhada pelos idosos apresentaram papel relevante na medida da percepção de suficiência de renda. Nesse sentido, é lícito concluir 
que o capital humano e os determinantes sociais relacionados aos idosos estudados são significativos, principalmente no tocante à situação de vida e na avaliação da condição socioeconômica verificada no momento da pesquisa.

Reforça-se, ainda, a importância do entendimento que os idosos possuiriam do atendimento ou não de suas necessidades básicas e humanas, pois, dependendo de como seja esse entendimento, a percepção de suficiência de renda poderia apresentar diferenças entre pessoas aparentemente semelhantes ou igualdade de percepções em pessoas teoricamente diferentes.

Pressupõe-se, assim, que não serão algumas medidas isoladas com a pretensão de erradicar a miséria absoluta que resolverão os problemas do atendimento das necessidades básicas e humanas dos indivíduos, principalmente quando chegam à velhice. A repetição ou manutenção de diferenças socioeconômicas, em nosso país, não garantirão aos novos idosos um futuro onde perce-

\section{Referências}

1. Popolo F. Características sociodemográficas y socioeconómicas de las personas de edad en América Latina. Santiago de Chile: Proyecto Regional de Población CELADE-FNUAP (Fondo de Población de las Naciones Unidas) Centro Latinoamericano y Caribeño de Demografía; 2001.

2. Rodrigues RAP, Kusumota L, Marques S, Fabrício SCC, Cruz IR, Lange C. Política nacional de atenção ao idoso e a contribuição da enfermagem. Texto Contexto Enferm 2007; 16(3):536-545.

3. Palloni A, De-Vos S, Pelaez M. Aging in Latin America and the Caribbean. Center for Demography and Ecology. University of Wisconsin. Madison. USA. Working Paper no 99-02, 2002. [acessado 2013 jun 15]. Disponível em: http://www.ssc.wisc.edu/ cde/cdewp/99-02.pdf

4. Crespo APA, Gurovitz E. A pobreza como um fenômeno multidimensional. RAE eletrônica 2004 marabr [acessado 2013 jun 15]; 1(2):[cerca de 4 p.]. Disponível em: http://www.scielo.br/pdf/raeel/v1n2 /v1n2a03.pdf

5. Blazer DG, Ericsson NS, Hybels CF. Perception of Unmet Basic Needs as a Predictor of Mortality Among Community-Dwelling Older Adults. Am J Public Health 2005; 95(2):299-304.

6. Gasparini L, Alejo J, Haimovich F, Olivieri S, Tornarolli L. Poverty among the elderly in Latin America and the Caribbean. Background paper for the World Economic and Social Survey 2007: The World Ageing Situation, Universidad de la Plata, 2007. [acessado 2013 jun 15]. Disponível em: http:// www.un.org/en/development/desa/policy/wess/ wess_bg_papers/bp_wess2007_lac.pdf bam sua renda como suficiente ou efetivamente capaz de suprir as necessidades de sua vida diária.

\section{Colaboradores}

JA Bento trabalhou na concepção, delineamento, análise, interpretação dos dados e redação do artigo; e ML Lebrão na coordenação da base de dados, revisão crítica e aprovação da versão final.

\section{Agradecimentos}

Agradecemos a Sr. a Fabíola Bof de Andrade, pósdoutoranda da Faculdade de Saúde Pública que, com sua competência e empenho, colaborou com correções para a melhoria deste trabalho.
7. Neef MN, Elizailde A, Hopenhayn M. Desenvolvimento e necessidades humanas. Reflexões sobre uma nova perspectiva. Há algo a acrescentar ao que já foi dito? In: Manfred A, Max-Neef. Tradução da Rede Viva. Desenvolvimento em escala humana: concepção, aplicação e reflexões posteriores. Blumenau: Edifurb; 2012. (Sociedade e ambiente, V. 6)

8. Barros RP, Mendonça R, Santos D. Incidência e natureza da pobreza entre idosos no Brasil. Rio de janeiro: IPEA; 1999. (Texto para discussão, N. 686).

9. Hammill M. Income poverty and unsatisfied basic needs. Economic Commission for Latin America and the Caribbean (ECLAC), Subregional Headquarters in Mexico. 15 December 2009. [acessado 2013 jun 15]. Disponível em: http://www.eclac.org/ publicaciones/xml/0/38190/L949.pdf

10. Barros RP, Carvalho M, Franco S. Pobreza multidimensional no Brasil. Rio de Janeiro: IPEA; 2006. (texto para discussão, n. 1227). [acessado 2013 jun 15]. Disponível em: http://www.ipea.gov.br/portal/ index.php?option $=$ com_content $\&$ view $=$ article \&id $=4372$ \& catid $=308$

11. Sen A. As Biko knew, powerlessness in actual lives is the hurdle justice must clear. The Guardian 2010; 23 mar. [acessado 2013 jun 15]. Disponível em: http:/ /www.guardian.co.uk/commentisfree/libertycentral/2010/mar/23/social-justice-philosophyfreedom?INTCMP $=$ SRCH

12. Kalache A. O mundo envelhece: é imperativo criar um pacto de solidariedade social. Cien Saude Colet 2008; 13(4):1107-1111 
13. Silva NN. Aspectos metodológicos: Processo de Amostragem. In: Lebrão ML, Oliveira Duarte, or ganizadores. SABE - Saúde, Bem-estar e Envelhecimento. O projeto SABE no município de São Paulo: uma abordagem inicial. Brasília: Organização PanAmericana de Saúde; 2003. p. 47-57. [acessado 2013 jun 15]. Disponível em: http://www.fsp.usp.br/sabe/ Extras/Livro_SABE.pdf

14. World Health Organization (WHO). The management of nutrition in major emergencies. Geneva: World Health Organization; 2000. [acessado 2013 jun 15]. Disponível em: http://whqlibdoc. who.int/ publications/2000/9241545208.pdf

15. Departamento Intersindical de Estatística e Estudo Socioeconômicos (DIEESE). Custo da Cesta Básica. [página na Internet]. [acessado 2013 jun 15]. Disponível em: http://turandot.dieese.org.br/bdcesta/ referencia.html

16. Engel E. Die Productions-und Consumtionsverhaltnisse des Königreichs Sachsen" in Zeitschrift des Statistischen Bureaus des Königlich-Sächsischen. Ministerium des Innern 1857; 8(9):1-54.

17. Chai C, Moneta A. At the origins of Engel curves estimation. The Papers on Economics and Evolution 2008; [documento da Internet]. April 30:1-15. [acessado 2013 Jun 15]. Disponível em: https:// papers.econ.mpg.de/evo/discussionpapers/200802.pdf

18. Townsend P. What is Poverty? An Historical Perspective. In: International Poverty Centre (UNDP). What is Poverty? Concepts and measures. Poverty in Focus 2006. Disponível em: http://www.ipcundp. org/pub/IPCPovertyInFocus9.pdf

19. Townsend P. The meaning of poverty. London School of Economics and Political Science. The British Journal of Sociology 2010; 61(Supl. 1):85-102, January. [acessado 2013 jun 15]. Disponível em: http://onlinelibrary.wiley.com/doi/10.1111/j.14684446.2009.01241.x/full

20. Instituto Brasileiro de Geografia e Estatística (IBGE). Pesquisa de Orçamentos Familiares: 2002-2003 primeiros resultados. Rio de Janeiro: IBGE; 2004. [acessado 2013 jun 15]. Disponível em: http://www.ibge. gov.br/home/estatistica/populacao/condicaodevida/ pof/2002/pof2002.pdf

21. Departamento Intersindical de Estatística e Estudos Socioeconômicos (DIEESE). Cesta Básica Nacional: salário mínimo nominal e necessário. [página na Internet]. [acessado 2013 jun 15]. Disponível em: http://www.dieese.org.br/analisecestabasica/ salarioMinimo.html

22. Brasil. Ministério da Previdência Social. Estatísticas: Anuário Estatístico da Previdência Social 2002. (Suplemento histórico). [página na Internet]. [acessado 2013 jun 15]. Disponível em: http://wwwl.previdencia. gov.br/suplemento/11_01_19_01_01.asp

23. Lebrão ML, Laurenti R. Saúde, bem-estar e envelhecimento: o estudo SABE no Município de São Paulo. Rev Bras Epidemiol 8(2):127-141.

24. Folstein MF, Folstein SE, Mchugh PR. A practical method for grading the cognitive state of patient for the clinical. J Psychiatr Res 1975; 12(3):189-198.

25. Icaza Mc, Albala C. Projeto SABE. Minimental State Examination (MMSE) del estúdio de dementia em Chile: análisis estatístico. Washington: Organização Pan-americana de Saúde (OPAS); 1999.
26. Rao JNK, Scott AJ. On Chi-squared tests for multiway contigency tables with cell proportions estimated from survey data. Ann Statist 1984; 12(1):46-60.

27. Archer KJ. Lemeshow S. Goodness-of-fit test for a logistic regression model fitted using survey sample data. The Stata Journal 2006; 6(1):97-105.

28. Reddy SG, Barnard College, School of International and Public Affairs. As Novas Estimativas Globais da Pobreza - Cavando mais Fundo num Buraco. International Policy. Centre for Inclusive Growth. Setembro 2008. One page number 65. [página na Internet]. [acessado 2013 jun 15]. Disponível em: http:// www.ipcundp.org/pub/port/IPCOnePager65.pdf

29. Marx K. O 18 de Brumário de Louis Bonaparte. Lisboa: Edições Avante; 2003.

30. Becker G. Investment in human capital: A theoretical analysis. The journal of political economy 1962; 70(5):9-49.

31. Moretto CF. O Capital humano e a ciência econômica: algumas considerações. Teor. Evid. Econ. 1997; 5(9):67-80.

32. Modigliani F. Life cycle, individual thrift, and the wealth of nations. The American Economic Review 1986; 76:150-171.

33. Educação, Comunicação e Arte na Defesa dos Direitos da Criança e do Adolescente (ECOAR). Brasília: Organização Internacional do Trabalho (OIT); 2007.

34. Milanovic B, Jovanovic B. Changes in the perception of the poverty line during the depression in Russia, 1993-96. World Bank Economic Review 1999; 13(3):539-559.

35. Litwin DSWH, Sapir MAEV. Perceived Income Adequacy Among Older Adults in 12 Countries: Findings From the Survey of Health, Ageing, and Retirement in Europe. Gerontologist 2009; 49(3):397-406.

36. Stoller MA, Stoller EP. Perceived income adequacy among elderly retirees. J Appl Gerontol 2003; 22(2): 230-251.

37. Toscano EV, Amestoy VA, Rosal RSD. Building financial satisfaction. Social Indicators Research 2006; 77(2):211-243.

38. Camarano AA. O idoso brasileiro no mercado de trabalho. Rio de janeiro: IPEA; 2001. (Texto para discussão, N. 830).

39. Debert GG. A reinvenção da velhice. Socialização e processos de reprivatização do envelhecimento. $1^{\text {a }}$ Edição. São Paulo: Editora da Universidade de São Paulo; 2004.

40. Zorzin PLG, Wajnman S, Turra CM. Previdência social e desigualdade racial no Brasil. In: Associação Brasileira de Estudos Populacionais (ABEP). Anais 2010 - ST - A demografia da previdência social: fontes de dados, aplicações e desafios. [página na Internet]. [acessado 2013 jun 15]. Disponível em: http://www.abep.nepo.unicamp.br/encontro2010/ docs_pdf/tema_9/abep2010_2033.pdf

Artigo apresentado em 03/04/2012

Aprovado em 30/05/2012

Versão final apresentada em 10/06/2012 